$$
\text { Submitted to J.Appl. Phys. }
$$

\title{
Acousto-optic modulation using a New Chlorotellurite Glass
}

I. Abdulhalim, J. Wang, C.N. Pannell, G. Wylangowski, D.N. Payne

\author{
Optoelectronics Research Centre \\ Optical Fibre Group \\ Southampton University \\ Southampton, SO9 $5 \mathrm{NH}$ \\ U.K.
}

We report results of initial tests on an acousto-optic modulator using a new chlorotellurite glass which has the composition $3 \mathrm{TeO}_{2}-2 \mathrm{ZnCl}_{2}$. This glass exhibits wider transparency range $(0.42-5.7 \mu \mathrm{m})$ than other tellurite glasses with approximately the same figures of merit. We obtained $80 \%$ diffraction loss and $65 \%$ diffraction efficiency at the wavelength 633 $\mathrm{nm}$, using an $80 \mathrm{MHz}$ piezoelectric transducer with dimensions $15 \mathrm{xl} \mathrm{mm}$ and using $1.5 \mathrm{~W}$, of RF power. This glass is able to convert to fiber form allowing for efficient in-fiber acousto-optic modulators to be built. 


\section{Introduction}

Materials which are particularly suitable for the construction of acousto-optic (AO) devices exhibit high acousto-optic interaction efficiency, low acoustic loss, high damage threshold, small temperature effects and good optical quality. ${ }^{1}$ In addition, they should be readily available and easy to fabricate, lap and polish. Many crystalline materials exhibit large acousto-optic figures of merit, however the difficulty in preparing mono-crystals in large size and quantities limits their suitability for AO devices. Glasses on the other-hand are isotropic by their nature, easy to fabricate in large quantities with good optical quality and it is usually possible to modify their transparency region without a significant sacrifice in their acousto-optic properties. The interest in tellurite glasses as acousto-optic materials originated from the fact the $\mathrm{TeO}_{2}$, the mother material of these glasses, exhibits relatively high acoustooptic figures of merit ${ }^{1}$ and is transparent in the range 0.33-5 $\mu \mathrm{m}$. A number of tellurite glasses have been investigated in the past ${ }^{2,3}$, however their practical transparency region is reduced to $0.45-2.7 \mu \mathrm{m}$. In this section we report on acousto-optic modulators made of a new glass fabricated at Southampton University. The glass composition is $3 \mathrm{TeO}_{2}-2 \mathrm{ZnCl}_{2}$ and it exhibits transparency in the range $0.42-5.7 \mu \mathrm{m}$, (Figure 1), wider than other reported ${ }^{2,3}$ AO tellurite glasses. Our interest in this glass system is mainly because we were able to $\mathrm{draw}^{4}$ an optical fiber from it which is transmitting in the infrared range. Therefore this glass will have a potential use for building in-fiber acousto-optic modulators. Such modulators have the advantage of low insertion loss, high damage threshold and negligible back reflection. They have applications for example in fiber sensors, and in Q-switching and mode-locking of fiber lasers. 


\section{Experimental}

The clorotellurite (CLT) glass composition we used is $3 \mathrm{TeO}_{2}-2 \mathrm{ZnCl}_{2}$, and was fabricated in our laboratories. ${ }^{4}$ The $\mathrm{TeO}_{2}-\mathrm{ZnCl}_{2}$ glass with $\mathrm{TeO}_{2} / \mathrm{ZnCl}_{2}$ ratio of 1.5 was prepared from dry mixtures of tellurium dioxide (Reagent grade, Aldrich) and zinc chloride (Analar grade, Aldrich). Quantities of the $\mathrm{TeO}_{2}$ and $\mathrm{ZnCl}_{2}$ powders were mixed in platinum or alumina crucibles of about $100 \mathrm{ml}$ capacity. Owing to the hydroscopic nature of $\mathrm{ZnCl}_{2}$, it was weighed first and covered with the $\mathrm{TeO}_{2}$ to prevent water absorption as much as possible. The mixture was then well mixed by mounting on a rotating lathe for a time period of about 30 minutes. The mix was subsequently transferred to an electrically-heated furnace, pre-heated to a temperature of $500^{\circ} \mathrm{C}$ to further dry the mix for two hours. The meltingfurnace temperature was then raised to $800^{\circ} \mathrm{C}$ for glass-melting, covered with a lid at the same time to reduce the vaporization. The melt was equilibrated for approximately 20 minutes after melting, with frequent shaking to achieve a bubble-free state. The melt was then cast into a mild-steel mould with internal dimensions of approximately $50 \mathrm{~mm}$ in length $\mathrm{x} 30 \mathrm{~mm}$ in width $\times 10 \mathrm{~mm}$ in thickness. Immediately after solidification, the mould was removed and the glass sample was put into a muffle furnace at $260^{\circ} \mathrm{C}$ and annealed for one hour then allowed to cool overnight.

The sample used to build the AOM was polished on its faces down to $\lambda / 4$ flatness with dimensions $16 \times 10 \times 6 \mathrm{~mm}$. The fabrication of an AOM from this glass was performed by Gooch \& Housego-UK, Ltd. The piezoelectric transducer is a $42 \mu \mathrm{m}$ thick $\mathrm{LiNbO}_{3}, \mathrm{Y}$-cut at $36^{\circ}$ to provide compressional wave at $80 \mathrm{MHz}$. A $1000 \AA$ thick gold layer deposited on one of the polished faces of the glass with the dimensions $6 \times 15 \mathrm{~mm}$ acts as the bottom 
electrode. On the opposite face a metallic absorber is attached in order to ensure travelling wave operation. The transducer is bonded directly to the gold using the technique of vacuum indium-cold-welding. A matching circuit was connected to the transducer to minimise the reflected RF power at resonance. The lateral dimensions of the transducer are $\mathrm{L}=15 \mathrm{~mm}$ long and $H=1 \mathrm{~mm}$ wide. To characterise the device, light from HeNe laser at $\lambda=633 \mathrm{~nm}$ was launched into a single mode fibre and at the distal end was recollimated using a quarter pitch GRIN lens. The output from a single mode fibre was used for two reasons. First, to obtain spatial filtering which is found to improve the diffraction efficiency. Secondly, to achieve a collimated Gaussian laser beam with small waist to demonstrate fast switching under pulsed operation. The collimated beam had a waist of $\omega_{b}=145 \mu \mathrm{m}$ measured using the knife edge technique.

\section{Results and Discussion}

Figure 2 shows the variation of the diffraction loss and the diffraction efficiency with the RF power under $\mathrm{CW}$ operation using a collimated HeNe laser beam at $633 \mathrm{~nm}$ with a beam radius of $145 \mu \mathrm{m}$. The diffraction loss is defined as the ratio between the light intensity in the $0^{\text {th }}$ order beam when the RF signal is ON to that when it is OFF. The diffraction efficiency is the ratio between the light intensity diffracted into the $1^{\text {st }}$ order beam to the transmitted intensity when the RF signal is OFF. The diffraction loss is usually higher than the diffraction efficiency because part of the light is diffracted to other diffraction orders since the AOM is not operating exactly in the Bragg regime.

In order to examine the speed of the device, the RF signal was modulated so as to 
provide $500 \mu$ s square pulses at $0.6 \mathrm{kHz}$ repetition rate and average $\mathrm{RF}$ power of $0.45 \mathrm{~W}$. The acoustic velocity was estimated from a measurement of the delay time between the RF pulse and the optical response as a function of the beam position from the transducer plane (figure 3) and found to be $v=3108 \pm 93 \mathrm{~m} / \mathrm{s}$. The value of $v$ obtained by this technique is very accurate (uncertainety is $3 \%$ ) because the position of the optical beam and the delay time are relative quantities measured with respect to fixed points in space and time domains respectively. Under these conditions we measured a rise time of 62 ns. The optical rise time $\tau_{r}$ is given by Maydan's formulae: $\tau_{r} \sim 1.3 \omega_{b} / v$, where $\omega_{b}=145 \mu \mathrm{m}$, is the beam radius. Using $\mathrm{v}=3108 \mathrm{~m} / \mathrm{s}$ yields $\tau_{\mathrm{r}}=60 \mathrm{~ns}$ in agreement with the measured value. Figure 4 shows the variation of the deflection angle $\delta$ with the acoustic frequency $v$, exhibiting the linear behavior $\delta=\lambda v / v$. The straight line is calculated using the value of $v$, estimated from figure 3 exhibiting good agreement with the measured values of $\delta$ within the experimental error. Figure 5 shows the variation of the diffraction efficiency with the RF frequency exhibiting a bandwidth of $\approx 20 \mathrm{MHz}$, which corresponds to $\approx 10$ mradians of angular width according to figure 4.

To determine the acousto-optic figures of merit, the experimental results of the diffraction efficiency in the $1^{\text {st }}$ order Bragg diffraction (Figure 2) were fitted to Gordon's, formulae:

$$
\eta=\sin \left(A\left(P_{r f}\right)^{0.5}\right)
$$

Here $A=\pi\left(\beta L M_{2} / 2 H\right)^{0.5} / \lambda$ with $\lambda$ being the optical wavelength in vacuum, $\beta$ is a factor which relates the acoustic power $\mathrm{P}_{\mathrm{a}}$ transferred to the $\mathrm{AO}$ medium to the RF electrical power $P_{r f}$ transferred to the transducer from the signal generator, $\beta=P_{a} / P_{r f}$, and $M_{2}$ $=\left(n^{6} p^{2} / \rho v^{3}\right)$ is the second AO figure of merit where $p$ is the photoelastic constant and $\rho$ is the 
density. The fitted curve in figure 2 was obtained with $A=0.761 \mathrm{~W}^{-0.5}$ which yields $\beta \mathrm{M}_{2}=$ $3.13 \times 10^{-15} \mathrm{sec}^{3} / \mathrm{kg}$. The factor $\beta$ was estimated to be $\beta \approx 0.8$ which then yields $\mathrm{M}_{2}=3.9 \times 10^{-15}$ $\sec ^{3} / \mathrm{kg}$. From this value, the acoustic velocity, the refractive index and the density of the CTL glass we estimated the effective photoelastic constant and the other figures of merit $M_{t}$ $=n^{7} p^{2} / \rho v$, and $M_{3}=n^{7} p^{2} / \rho v^{2}$. The figures $M_{1}, M_{2}$, and $M_{3}$ represent scanning capability, diffraction efficiency and number of resolvable spots respectively. ${ }^{1}$ The values obtained for the figures of merit are believed to be lower than the true values. This is because at the frequency of $80 \mathrm{MHz}$, the device is not operating completely in the Bragg regime. Some light, although being weak, was observed in other diffraction orders. To distinguish between Bragg and Raman-Nath regimes, the parameter $\mathrm{Q}=2 \pi \lambda \mathrm{L} / \mathrm{n} \Lambda^{2}$, is usually introduced where $\Lambda$, is the acoustic wavelength. The limit $\mathrm{Q}>1$ places the device in the Bragg regime. In our case $\Lambda=38.85 \mu \mathrm{m}$ at $80 \mathrm{MHz}$, which yields $\mathrm{Q} \approx 19$, and so the device is operating mainly but not completely in the Bragg regime. This is manifested by the fact that the diffraction loss is higher than the diffraction efficiency (figure 2). Effectively this lowers the optimum value of the diffraction efficiency to be less than $100 \%$. Therefore the value of $\mathrm{M}_{2}$ estimated by fitting the results to equation (1) is the minimum value. A more accurate estimate of the figures of merit can be performed using the technique of Dixon and Cohen. ${ }^{6}$

Table I summarises the relevant parameters of the CLT glass as an AO material. The values of $M_{1}, M_{2}$, and $M_{3}$ indicate that this glass is a highly promising $A O$ material for modulators and deflectors which together with the high acoustic velocity allows for fast switching and scanning. It should be noted here that at high frequencies, the diffraction efficiency into the $1^{\text {st }}$ order Bragg diffraction should improve. This is because the acoustic velocity is high and therefore the acoustic wavelength $\Lambda$ is larger for the same frequency, so 
that if the transducer length is insufficient for low frequencies (tens of $\mathrm{MHz}$ ), additional diffraction peaks can appear due to Raman-Nath type diffraction. It should be noted here that tellurite glasses ${ }^{2,3}$ exhibit low acoustic loss because of their short relaxation time $\tau_{\mathrm{p}}$ of the lattice phonons. The ultrasonic loss at frequencies up to few hundreds of $\mathrm{MHz}$ is proportional to $\tau_{\mathrm{p}}$ and increases quadratically with the frequency. It can be described according to Woodruff-Ehrenreich equation?:

$$
\alpha=4 \pi^{2} c T \gamma^{2} v^{2} \tau_{\mathrm{p}} / 3 \rho v^{3} .
$$

Here $\mathrm{c}$ is the specific heat, $\gamma$ is the mean Gruneisen parameter and $\mathrm{T}$ is the temperature. We have not measured the acoustic loss directly, but we did not observe any significant decrease of the diffraction efficiency as the optical beam position from the transducer was changed over a distance of $\approx 1 \mathrm{~cm}$. This indicates that the CLT glass exhibits low ultrasonic absorption at $80 \mathrm{MHz}$. This is not surprising, however, since the glass network-forming oxide $\mathrm{TeO}_{2}$, which exhibits low ultrasonic absorption $(\alpha<1 \mathrm{~dB} / \mathrm{cm})$ at $80 \mathrm{MHz}$, constitutes the major part of the composition. Low acoustic absorption means that higher frequency operation of AOMs made of CLT glass is possible. This increases the deflection angle, the bandwidth, the resolving power and the diffraction efficiency.

In conclusion, we have found that the chlorotellurite glass based on $\mathrm{TeO}_{2}-\mathrm{ZnCl}_{2}$ systems can be used as an acousto-optic material. The properties of this glass combined with its transparency both in the visible and the mid-infrared, nontoxity, ease of fabrication, optical quality and isotropic nature, make it attractive for acousto-optic device applications. 
Acknowledgements: This work was supported by a U.K. government (DTI) LINK project in collaboration with Gooch \& Housego Ltd. The ORC is a U.K. government SERC sponsored interdisciplinary research centre. We would like to thank Mr. D. Moreau, and Mr. G. Jones for useful interactions and discussions. 


\section{References}

${ }^{1}$ L. N. Magdich and V. Ya. Molchanov, Acousto-optic Devices and their Applications (Gordon \& Breach Science Publishers, London, 1989).

${ }^{2}$ T. S. Izumiatani, Optical Glass (American Institute of Physics, Translation Series, New York 1986), pp 177-179.

${ }^{3}$ T. Yano, A. Fukumoto and A. Watanabe, J. Appl. Phys. 42, 3674 (1971).

${ }^{4} \mathrm{~J}$. Wang, Novel Multicompnent Glasses and Fibres for Fibre-optic Devices and Systems, $\mathrm{PhD}$ thesis (Univesity of Southampton, March 1993).

${ }^{5}$ D. Maydan, IEEE J.Quant.Elect. QE-6, 223 (1970).

${ }^{6}$ R. W. Dixon and M. G. Cohen, Appl.Phys.Lett. 8, 205 (1966).

${ }^{7}$ T. O. Woodruff and H. Ehrenreich, Phys.Rev. 123, 1553 (1961). 


\section{Figure Captions}

Figure 1. Transmittance spectrum of the chlorotellurite glass using a $2 \mathrm{~mm}$ thick sample. Fresnel reflections were not ruled out in this spectrum.

Figure 2. The variation of the measured diffraction loss and efficiency with the RF power. The solid line is the fitted diffraction efficiency using equation (1).

Figure 3. The delay time between the applied RF pulse and the optical response as a function of the optical beam position from the transducer. The RF pulse width is $500 \mu \mathrm{s}$, the repetition rate is $0.6 \mathrm{kHz}$ and the average $\mathrm{RF}$ power is $0.45 \mathrm{~W}$.

Figure 4. Variation of the measured deflection angle with the RF frequency under CW operation using $0.45 \mathrm{~W}$ RF power. The solid curve is calculated using the value of the acoustic velocity determined from figure 3 .

Figure 5. Diffraction efficiency versus the RF frequency under CW operation using 1.5 W RF power. 


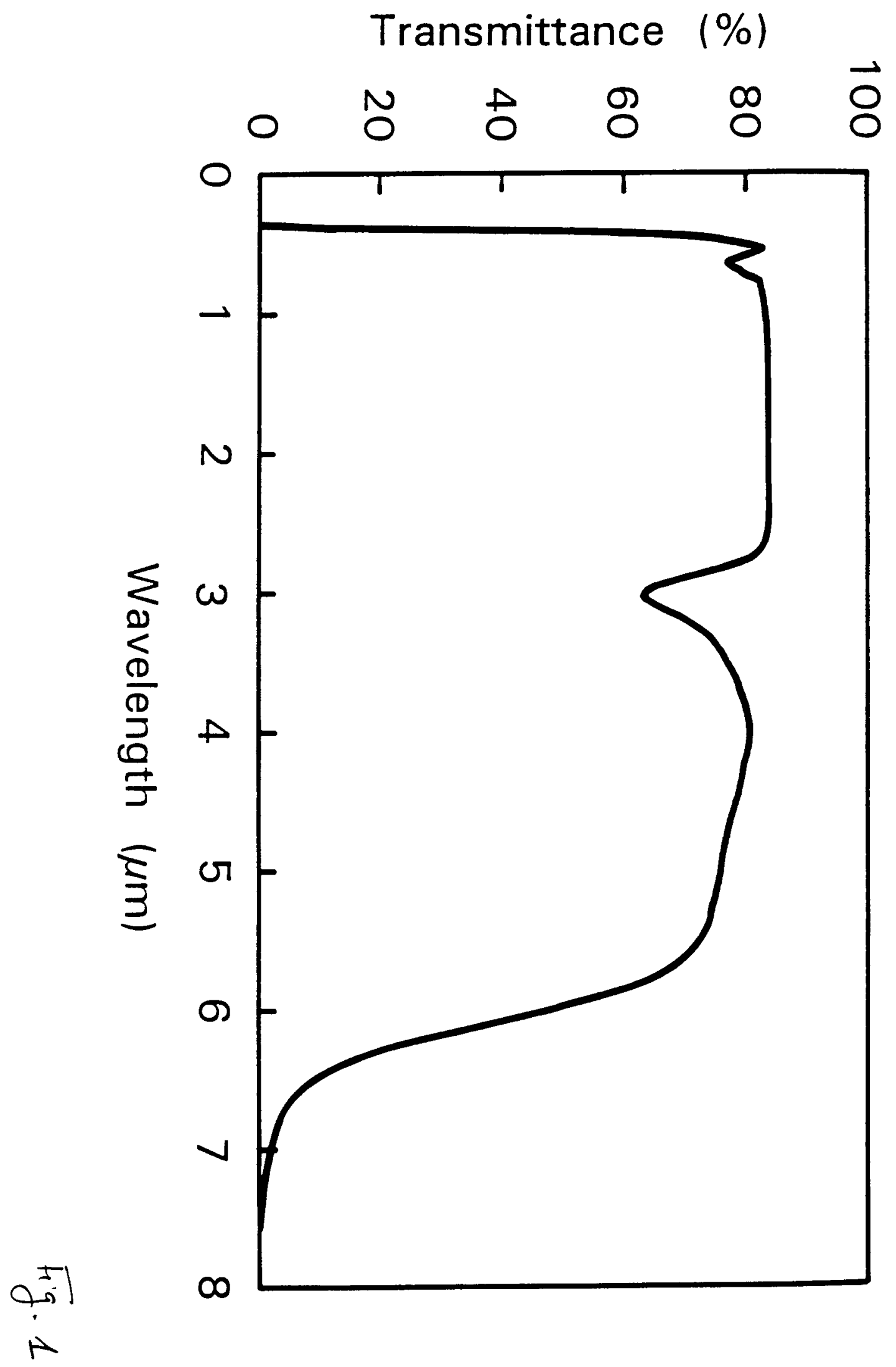




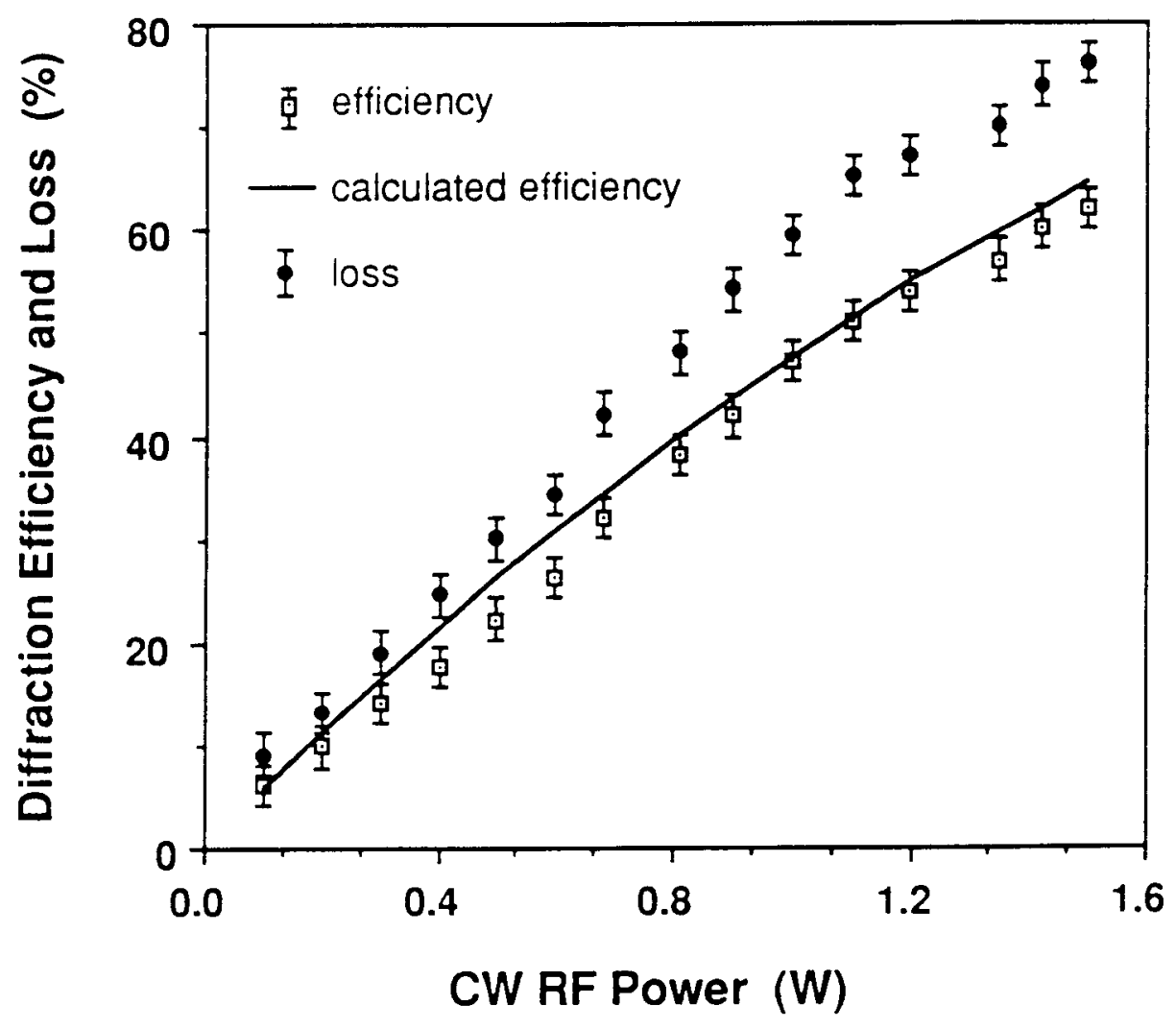

Fig. 2 


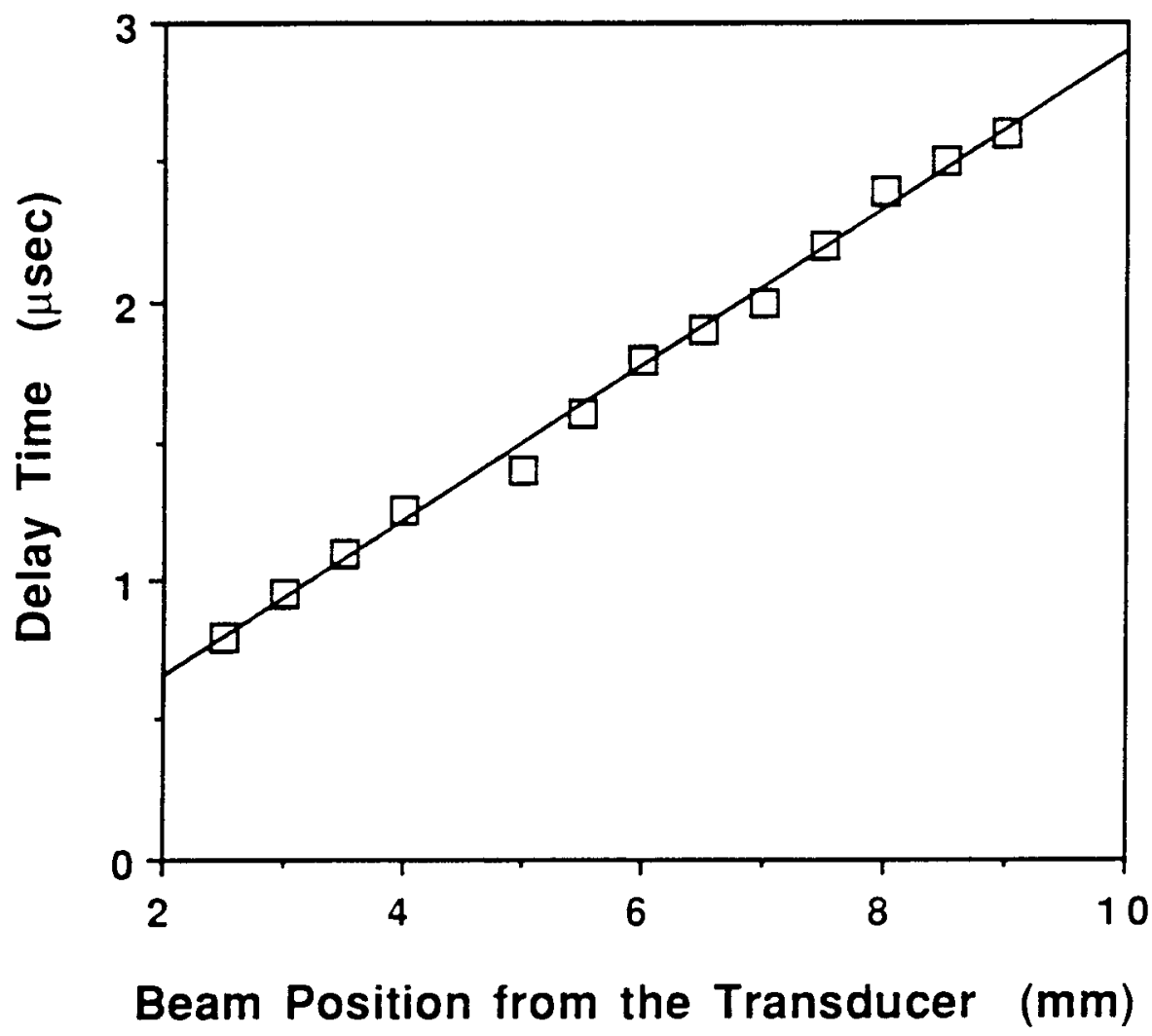

Fig. 3 


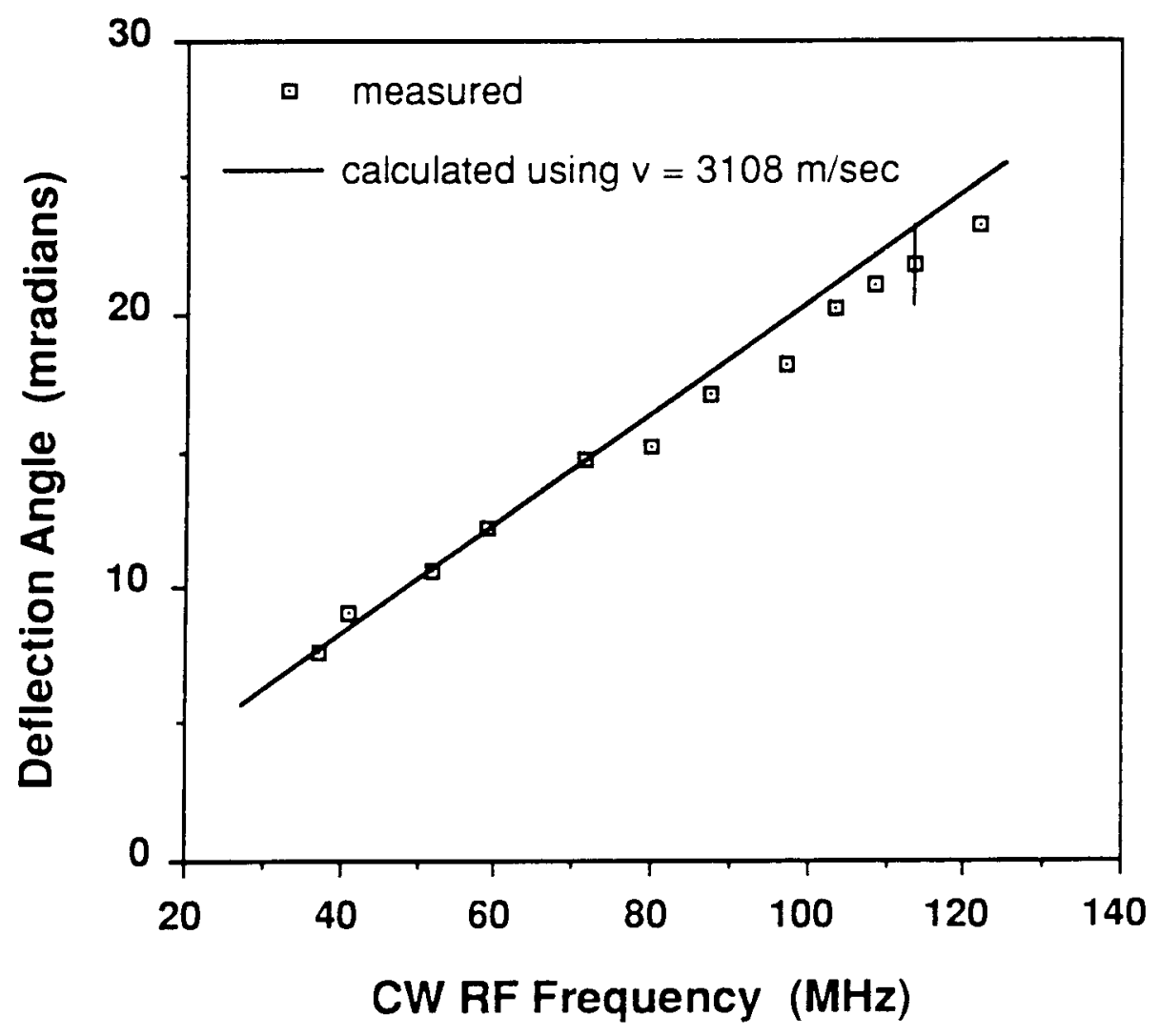

Fig. 4 


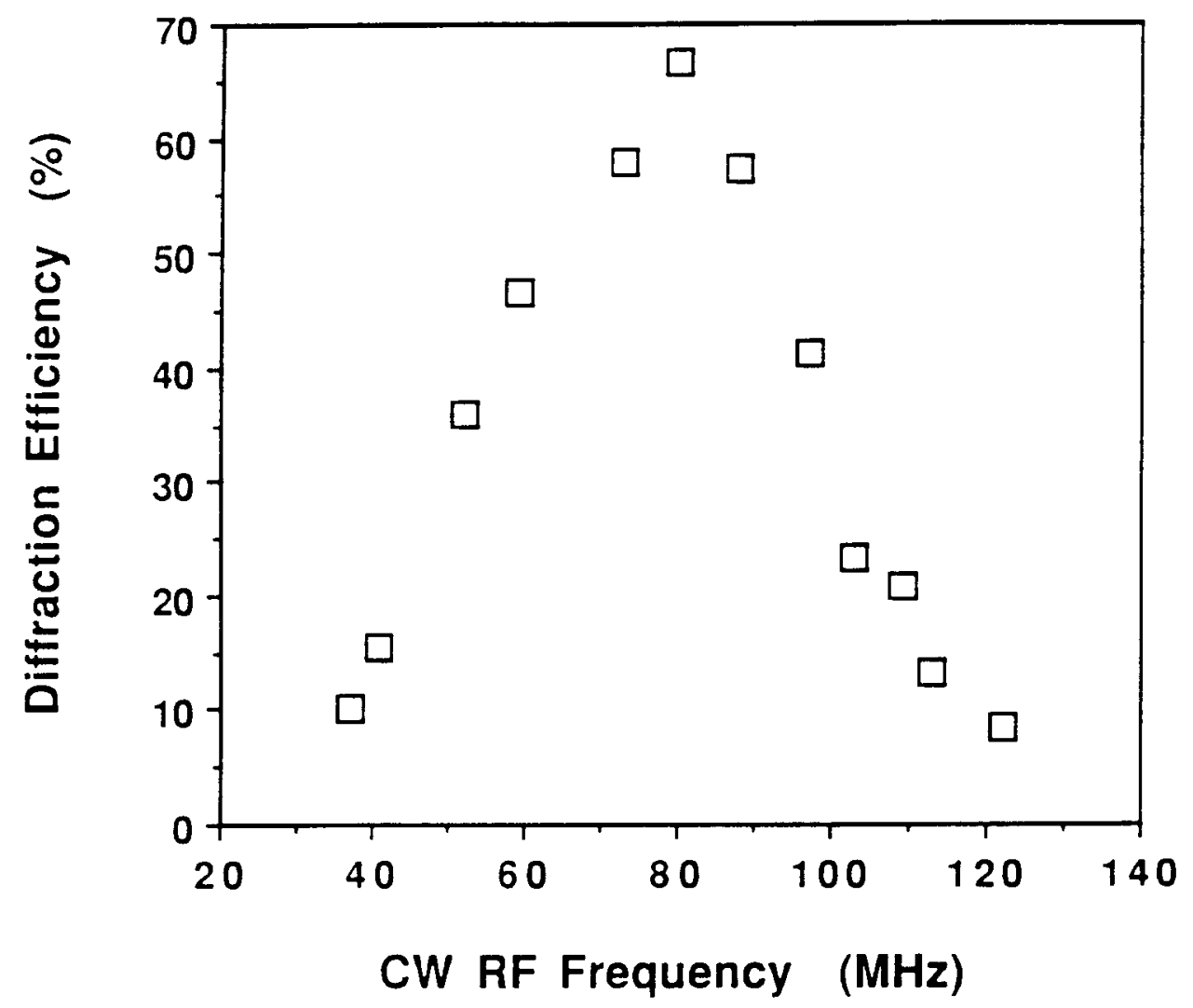

Fig. 5 


\section{Tables Caption}

Table I. Physical parameters and estimated acousto-optic parameters of the CLT glass.

\begin{tabular}{ll} 
Optical transmission region $(\mu \mathrm{m})$ & $0.42-5.7$ \\
Refractive index $(633 \mathrm{~nm})$ & 2.0 \\
Density $(\mathrm{g} / \mathrm{cc})$ & 230 \\
Glass transition temperature $\left(\mathrm{C}^{\circ}\right)$ & 230 \\
Acoustic attenuation at $80 \mathrm{MHz}(\mathrm{dB} / \mathrm{cm})$ & $<1.0$ \\
Longitudinal acoustic velocity $(\mathrm{m} / \mathrm{s})$ & 3108 \\
Young's $\mathrm{Modulus}\left(\mathrm{x} 10^{10} \mathrm{~N} / \mathrm{m}^{2}\right)$ & 4.25 \\
Pockel's coefficient & 0.09 \\
$\mathrm{M}_{1}\left(\mathrm{x} 10^{-7} \mathrm{sm} / \mathrm{kg}\right)$ & 0.76 \\
$\mathrm{M}_{2}\left(\times 10^{-15} \mathrm{~s}^{3} / \mathrm{kg}\right)$ & 3.9 \\
$\mathrm{M}_{3}\left(\times 10^{-10} \mathrm{~s}^{2} \mathrm{~m} / \mathrm{kg}\right)$ & 0.24 \\
\hline
\end{tabular}

